\title{
Effect of Osteopathic Manipulative Therapy on Generalized Anxiety Disorder
}

Lindsay Dixon, BSc (Hons), DOMP, DScO; Kathryn Fotinos, BSc (Hons); Emilia Sherifi, BSc (Hons); Sachinthya Lokuge, BSc (Hons); Alexa Fine, BSc (Hons); Melissa Furtado, BSc (Hons); Leena Anand, MA; Katharine Liberatore, DOMP; Martin A. Katzman, MD

From the S.T.A.R.T. Clinic for Mood and Anxiety Disorders

in Toronto, Ontario (Mss Dixon, Fotinos, Sherifi, Fine,

Furtado, and Anand, Mr Lokuge, and Dr Katzman); the Department of Psychology at Lakehead University in

Thunder Bay, Ontario (Dr

Katzman); the Northern Ontario School of Medicine in Sudbury (Dr Katzman); the Department of Psychology at the Adler Graduate

Professional School in Toronto, Ontario (Dr Katzman); and the Canadian

College of Osteopathy in North York, Ontario (Ms Dixon and Ms Liberatore).

Financial Disclosures: None reported.

Support: None reported.

Address correspondence to Martin A Katzman, MD, S.T.A.R.T. Clinic for Mood and

Anxiety Disorders, 32 Park Rd, Toronto, Ontario, Canada M4W $2 N 4$.

Email: mkatzman@ startclinic.ca Submitted February 27, 2019; revision received May 21, 2019; accepted June 24, 2019.
Context: Traditional management options for generalized anxiety disorder (GAD) have produced low remission rates. As a result, the medical community has turned to complementary and alternative medicine for adjunctive treatment.

Objective: To investigate the efficacy of adjunctive osteopathic manipulative therapy (OMTh; manipulative care provided by foreign-trained osteopaths) in individuals with GAD.

Methods: This open-label, nonrandomized, black-box study took place at a tertiary care mental health clinic in Toronto, Canada. Adult outpatient participants aged 18 to 65 years with a primary diagnosis of moderate-severe GAD (HAM-A score of $\geq 20$ ) with or without comorbidities were enrolled in the study between June 2014 and January 2015. Patients who qualified and completed the study received 5 individually tailored OMTh sessions over the course of 8 to 9 weeks. A diagnostic psychiatric assessment (Mini International Neuropsychiatric Interview version 6.0.0) was conducted to confirm diagnoses, along with physician-administered and self-reported measures of anxiety, including the Hamilton Anxiety Scale (HAMA), the Beck Anxiety Inventory, and the Intolerance for Uncertainty Scale.

Results: Significant reductions in total HAM-A scores after OMTh were observed $(P<.0001)$. Significant reductions in total Intolerance for Uncertainty Scale scores were also observed $(P<.0001)$. Beck Anxiety Inventory scores were not found to change significantly with OMTh. Response (defined as $50 \%$ reduction of symptoms) and remission (defined as HAM-A score of $\leq 7$ ) rates were found to be $62 \%$ and $26.9 \%$, respectively.

Conclusion: Osteopathic manipulative therapy may be a valuable adjunct to conventional therapy in patients with GAD, thus warranting further investigation using double-blind procedures.

J Am Osteopath Assoc. 2020;120(3):133-143

doi:10.7556/jaoa.2020.026

Keywords: generalized anxiety disorder, mental health, osteopathic manipulative therapy

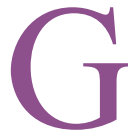
eneralized anxiety disorder (GAD) is a chronic, often lifelong, disorder and a global health concern causing enormous economic and social burden. ${ }^{1}$ It is characterized by cognitive symptoms that include excessive anxiety, uncontrollable worry, and difficulty concentrating, which often manifest in physical symptoms such as restlessness, fatigue, irritability, muscle tension, and sleep disturbances. ${ }^{2}$ This 
disorder is considered among the most costly and least successfully treated anxiety disorders because of its high comorbidity rates with other mood and anxiety disorders. ${ }^{3}$ Generalized anxiety disorder poses significant impairments to quality of life and increases risk for suicidality, ${ }^{4}$ hence the renewed urgency to investigate novel methods for enhancing treatment outcomes for patients with GAD.

Standard care treatment options for patients with GAD include both pharmacologic and psychological options, both of which focus on addressing the individual's neurochemistry and emotional dysregulation. ${ }^{2,5}$ Nevertheless, remission rates remain low, with significantly less than $50 \%$ of individuals with GAD reporting remission following standard care. ${ }^{2}$ As a result, the medical community has placed greater emphasis on exploring new adjunctive treatment options, specifically in the complementary and alternative medicine fields. ${ }^{6,7}$

Osteopathic manipulative therapy (OMTh; manipulative care provided by foreign-trained osteopaths) is characterized as a holistic, hands-on therapy emphasizing that the body systems are interconnected and function as a single unit. ${ }^{8}$ The musculoskeletal system has been viewed as having a vital influence on overall body system health and functioning, with impaired mobility of the body potentially disrupting an individual's overall health and contributing to disease, including mental illness. ${ }^{8,9}$ Hands-on touch, which is integral to OMTh, ${ }^{10}$ has been demonstrated to activate $\beta$ and $\mathrm{C}$ tactile receptors that contribute to a release of oxytocin, thereby causing a downregulation of the hypothalamuspituitary-adrenal (HPA) axis. ${ }^{11,12}$ The release of oxytocin contributes to systemic effects, including decreased heart rate and blood pressure, decreased sensitivity to pain stimuli, and global anxiolytic effects. ${ }^{12}$ Furthermore, OMTh targets structures related to the autonomic nervous system, including structural and neurovascular pathways that disrupt homeostasis and self-regulation, ${ }^{8,9}$ ensuring that these systems are free from tension in order to support optimal functioning. ${ }^{13-15}$

Osteopathic manipulative therapy also includes evaluation of fascia. ${ }^{16}$ The fascial continuity of the body plays a major role in the communication and integration of all bodily systems. ${ }^{13,17}$ The musculoskeletalsystem, which includes the fascia, interacts with the endocrine, neurovascular, and immune systems through mechanical links and feedback systems. ${ }^{10}$ When fascia is placed under prolonged tension, the mobility and function of the structures it binds to and all materials that pass through it may be disrupted. ${ }^{15}$ The disruption in mobility and function of these binding structures may lead to increased afferent sensory and nociceptive feedback to the brain, thereby influencing emotional response. ${ }^{13}$ Osteopathic manipulative therapy serves to address the physical tension in the body in an effort to reduce the afferent sensory and nociceptive feedback processes that dysregulate emotional response.

Generalized anxiety disorder has been associated with abnormal neurochemistry and digestion, as observed in the gut-brain axis, which refers to the crosstalk between the enteric nervous systems of the gut and the brain. ${ }^{18-20}$ Through this relationship, the gut may influence emotional response, and conversely, the mind may influence digestive and immune health. Osteopathic manipulative therapy focuses on enabling a free pathway from the neurovascular supply to the intestines and liver, including fascial connections, ${ }^{21}$ to support digestion and immune function. Facilitating the mobility of the abdominal fascia and the lumbar spine may decrease nonspecific pain and tension in those regions via influence on the mechanoreceptors and on the afferent vagal pathway, ${ }^{13}$ which may ameliorate the symptoms often associated with GAD.

Osteopathic manipulative therapy is gaining momentum with research that examines cerebral blood flow, ${ }^{22}$ cortical plasticity, ${ }^{23}$ immune function, ${ }^{24}$ heart rate variability, ${ }^{25}$ muscle tension, ${ }^{26}$ and common physical comorbidities of GAD, such as migraines, ${ }^{27,28}$ irritable bowel syndrome, ${ }^{29}$ and back pain. ${ }^{30}$ Furthermore, studies have reported a reduction in depressive symptoms, ${ }^{31}$ state-trait anxiety, ${ }^{28}$ and reduced muscle tension as a result of components of OMTh, including 
myofascial work, spinal mobilization, and cranial techniques. ${ }^{26}$ Similar techniques were used to reduce sleep disturbance, ${ }^{32}$ headaches, ${ }^{33}$ migraines, ${ }^{28}$ and pain, ${ }^{26,28}$ all of which are secondary symptoms observed in individuals with GAD.

Nevertheless, the literature on OMTh in the management of mental illness is sparse and lacks generalizability. Given the dearth of information about using OMTh as management for anxiety disorders, specifically GAD, this study was undertaken to investigate OMTh as an adjunctive treatment for individuals with a diagnosis of GAD. The authors hypothesized that an all-encompassing individualized approach in OMTh would reduce the severity of symptoms associated with GAD.

\section{Methods}

The study ran from June 2014 to January 2015 and patients were recruited through clinic referrals and advertisements. A foreign-trained osteopath (L.D.) contacted interested participants to inform them of the study details and schedule a time for a brief telephone assessment, which served as a screening tool to determine eligibility based on the inclusion criteria. A member of the research personnel (M.F. and L.A.) conducted each of the phone screening assessments, which were a semi-standardized assessment tool created by the study psychiatrist (M.A.K.).

\section{Participants}

Eligibility for this study consisted of outpatients who had been referred to a tertiary care psychiatry clinic in Toronto, Canada, were aged 18 to 65 years with a primary diagnosis of GAD, and had not achieved remission after a minimum of 8 weeks of standard treatment. ${ }^{2}$ Participants received their current treatments for the duration of the study and were informed that their decision to participate in the study would not influence future treatment opportunities. Those meeting inclusion criteria and not meeting exclusion criteria (Figure 1) were invited to provide informed consent. Ethics approval from the Canadian Memorial Chiropractic
Ethics Board was obtained in April 2014, and continuous patient recruitment began in June 2014 via clinic referrals and advertisements. Recruitment and data collection were completed in March 2015.

\section{Setting}

The S.T.A.R.T. (Stress Trauma Anxiety Rehabilitation Treatment) Clinic for Mood and Anxiety Disorders is a private practice offering psychiatric, psychological, psychotherapeutic, naturopathic, and holistic services. The room that was used for the osteopathic assessments and OMTh was approximately $14 \times 18$ feet with a window, light dimmer, air conditioning unit, and space heater to keep lighting and temperature at a comfortable level. The table used for OMTh was a nonhydraulic massage table.

\section{Procedures}

This was an open-label, black-box, before-and-after study design with no control group; every participant received OMTh. The clinicians (M.A.K. and L.D.) were not blinded. Participants were administered outcome measures at screening (visit 1), baseline (visit 2), and follow-up visits (visits 3-7). The secondary efficacy end points were the mean changes from before to after OMTh on the BAI ${ }^{37}$ and the IUS. ${ }^{38}$ Participants were assessed at the initial screening visit using the Mini International Neuropsychiatric Interview (MINI) 6.0.0 $0^{34}$ to screen for a primary diagnosis of GAD and to rule out other primary mood and anxiety disorders. At screening, participants were further assessed using the physician-administered Hamilton Anxiety Rating Scale (HAM-A) ${ }^{35}$ and Hamilton Depression Rating Scale (HAM-D). ${ }^{36}$ The treating psychiatrist (M.A.K.) conducted all assessments and the screening visit (visit 1), which included a brief medical and physical examination. Participants were also given a package of self-reported questionnaires $^{37,38}$ to confirm safety and acceptability. Each participant received 5 sessions of OMTh during the period of 8 to 9 weeks. Visit 2 was scheduled for 1 week after the initial screening visit and was the 


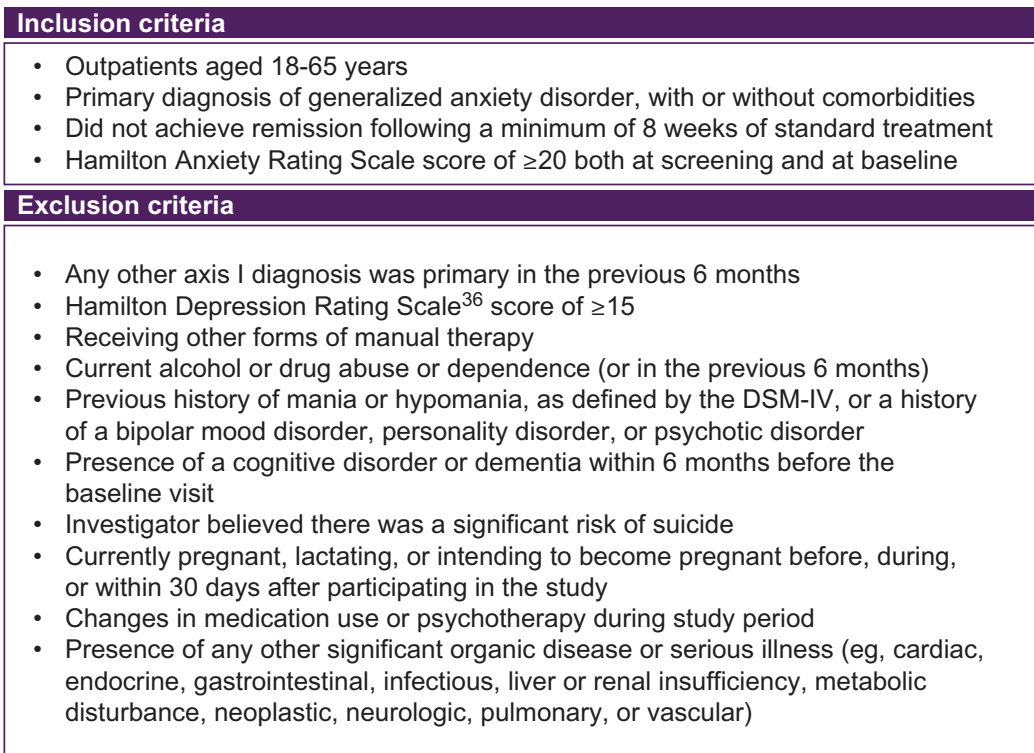

Figure 1.

Inclusion and exclusion criteria for study participants. Abbreviations: DSM-IV, Diagnostic and Statistical Manual of Mental Disorders, Fourth Edition.

baseline OMTh assessment. Visits 2, 3, and 4 (the first, second, and third OMTh appointments) were scheduled weekly, plus or minus 3 days to accommodate scheduling conflicts. Visits 5 and 6 (the fourth and fifth OMTh appointments) were scheduled to occur biweekly, plus or minus 3 days. Reassessment of the primary and secondary measures took place immediately before each OMTh appointment. At visit 7, scheduled 1 week after visit 6 (plus or minus 3 days) the psychological assessments were readministered.

A foreign-trained osteopath (L.D.) performed the OMTh. The psychiatrist (M.A.K.) administered the MINI, the HAM-A, and the HAM-D, as well as the brief medical and physical examinations. Two selfreported questionnaires (Beck Advisory Index [BAI] and Intolerance of Uncertainty Scale [IUS]) were used as secondary outcome measures, distributed to participants, and later collected by clinic research associates.

Participants were administered outcome measures at screening (visit 1), baseline (visit 2), and follow-up visits (visits 3-7). The primary efficacy end point was the mean change from before to after OMTh on the HAM-A. ${ }^{35}$ The secondary efficacy end points were the mean changes from before to after OMTh in the $\mathrm{BAI}^{37}$ and the IUS. ${ }^{38}$

\section{Instruments}

MINI Version 6.0.0

A semistructured, physician-administered diagnostic interview, the MINI, was developed to assist clinicians (ie, osteopaths, physicians, and psychologists) in the assessment and tracking of Axis-I disorders based on diagnostic criteria from the Diagnostic and Statistical Manual of Mental Disorders, Fourth Edition $(D S M-I V) .{ }^{34}$ It has good reliability and validity compared with the Structured Clinical Interview for DSM-IV Axis I Disorders, and is preferred because of a shorter administration time. The MINI has also demonstrated high interrater reliability. ${ }^{34}$

\section{Hamilton Anxiety Rating Scale}

The change in overall anxiety severity was measured using a 14-item physician-administered scale, the HAM-A, which has demonstrated high interrater reliability and validity. ${ }^{35,39}$ Each item was scored on a scale of 0 (not present) to 4 (severe), with a total score 
range of 0 to 56. A score less than 17 indicates mild anxiety, a score of 18 to 24 indicates mild to moderate anxiety, and a score of 25 to 30 indicates moderate to severe anxiety. ${ }^{35}$ Response to OMTh intervention using the HAM-A scale was considered to be a reduction of $50 \%$ of symptoms, and remission was defined as a score of less than or equal to $7 .{ }^{40}$

\section{Beck Anxiety Inventory}

The physiological symptoms of anxiety were measured using a 21-item self-reported scale, the BAI, which has demonstrated high internal consistency $(\alpha=.92)$ and good test-retest reliability ( $r=0.75){ }^{37,38,41}$ The BAI was completed in 5 to 10 minutes. Respondents rated how much they had been bothered by each of the 21 symptoms over the past week on a 4-point severity scale ranging from 0 to 3. The total score was derived by summing the ratings from all 21 questions and could range from 0 to 63 . Responses on these questionnaires are summed to produce a total anxiety severity score ( $0-7$, minimal; 8-15, mild; 16-25, moderate; 26-63, severe). ${ }^{37}$

\section{Intolerance of Uncertainty Scale}

An individual's inability to tolerate uncertain events, which is thought to be a defining feature of GAD and considered to be a central component of worry, was measured using the IUS. ${ }^{42}$ This scale is a 27 -item self-reported questionnaire that has demonstrated good test-retest reliability $(r=0.78)$ and has excellent internal consistency. ${ }^{43,44}$ Additionally, a high correlation with baseline scores exists between the HAM-A and the IUS. ${ }^{42,45}$ Participants are instructed to rate the extent to which the statements are characteristic of them on a scale of 1 to 5 , where 1 is not at all characteristic and 5 is entirely characteristic of them. Higher scores are associated with higher intolerance for uncertainty and increased anxiety severity.

\section{Osteopathic Manipulative Therapy}

A thorough examination of posture and mobility, and palpation testing of the spine, joints, cranial bones, dura, fascia, and viscera was used to determine the priorities and sequence for OMTh. ${ }^{46}$ Using OMTh, the whole body was assessed and treated, which allowed for structures related to emotional control, including the limbic, autonomic, and endocrine systems, to be evaluated. Alterations in tissue tone, texture, and restrictions in mobility and range of motion were noted for each participant. The most severe tissue restrictions were considered primary and given priority for OMTh. ${ }^{8,46}$ Soft tissue/fascia, visceral, cranial, osteoarticular techniques were applied based on the individual needs observed in each participant. ${ }^{16,21,46,47}$

Participants' unique medical and physical histories were also taken into consideration during the assessment to help determine best OMTh techniques. The black-box ${ }^{48}$ study design allowed for each patient to receive OMTh tailored to his or her individual dysfunctions, as per the initial assessment. The black-box study design also offered greater flexibility in terms of applying more comprehensive OMTh techniques (eg, muscle energy, myofascial release direct/indirect, cranial techniques, balanced ligamentous tension technique, and visceral manipulation) that aligned with the unique needs of the individual. ${ }^{48}$ Despite having poor internal validity because of the irreproducibility of individual components of OMTh, the black-box design demonstrates strong external validity, which is suggestive of generalizability to a larger population. ${ }^{49}$ This blackbox study design is also used to test the overall efficacy of OMTh in a clinical setting, which is reflective of the actual clinical practice of OMTh. ${ }^{48}$

The initial OMTh appointment was 1.5 hours, with successive appointments lasting 60 minutes, including a 15-minute reassessment. The 15-minute reassessment of tissue tone, symmetry, texture, and restrictions in mobility and range of motion occurred before each OMTh appointment. All OMTh assessment findings and observations during therapy were recorded on a standard form for each participant.

\section{Statistical Analysis}

SPSS Statistics Version 24 (IBM) was used to conduct statistical analyses. The Shapiro-Wilk test for normality was performed and yielded a normal distribution of 
data. To test the hypothesis that OMTh would have a mediating effect on GAD symptoms, a series of paired sample $t$ tests in observed cases (OC) and last observed carried forward (LOCF) analyses were conducted. The OC dataset included participants who completed all 7 visits. The LOCF analysis was conducted for participants who received OMTh at least 1 time and had at least 1 postbaseline evaluation. The LOCF was used to account for the participants who dropped out of the study and included participants who either completed the study or did not complete the study. The level of significance for all statistical analyses was set at $P \leq .05$.

\section{Results}

Twenty-six participants ( 6 men, 20 women) with a mean (SD) age of 41.4 (5.09) years completed the study. One patient was withdrawn before beginning OMTh because of an underlying unrelated medical condition, and 2 participants dropped out because of scheduling difficulties. Some of the relevant baseline physical health characteristics included low back pain (69\%), muscle tension (92\%), irritable bowel syndrome (65\%), and insomnia/sleep disturbance (92\%). Psychiatric comorbidities are depicted in Figure 2.

A significant effect was found in an OC analysis between mean scores on the HAM-A (primary outcome measure) between visit 1 and visit 7 , $\left(t_{25}=14.77, \quad P<.0001, \quad 2\right.$-tailed, Cohen $d=2.79$ ). Specifically, participants' anxiety decreased significantly from visit 1 (mean [SD], 26.19 [3.88]) to visit 7 (11.29 [6.11]), as measured by the HAM-A. Similar outcomes in differences in mean (SD) scores on the HAM-A were observed in a LOCF analysis from visit

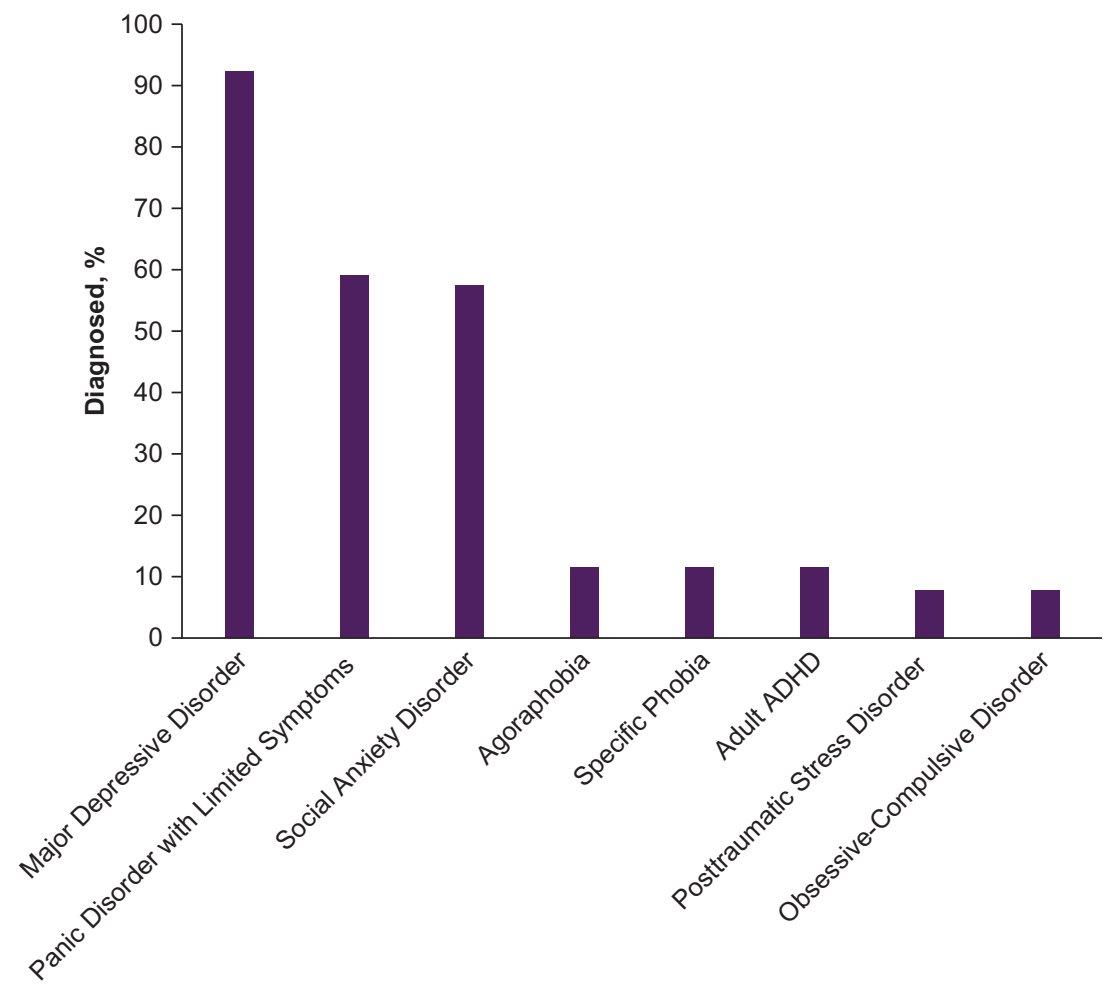

Comorbid Psychiatric Disorder

Figure 2.

A breakdown of the percentage of patients with generalized anxiety disorder who had a comorbid psychiatric disorder. Abbreviation: ADHD, attention deficit/hyperactivity disorder. 
1 to visit $7\left(t_{25}=14.68, P<.0001,2\right.$-tailed, Cohen $d=2.73$ ), where mean (SD) anxiety scores decreased significantly from visit $1(26.25$ [ 3.74]) to visit 7 (12.39 [0.14]). Ultimately, $62 \%(n=16)$ of the sample demonstrated a $50 \%$ reduction in HAM-A symptoms from baseline to last visit. Seven participants achieved remission, as defined by a HAM-A score of less than or equal to 7 .

Significant results were also observed in an OC complete analysis on IUS scores from visit 1 to visit 7, $\left(t_{25}=4.23, \quad P<.0001, \quad 2\right.$-tailed, Cohen $\left.\quad d=0.67\right)$. Specifically, mean (SD) IUS scores decreased significantly from visit 1 (80.08 [18.32]) to visit 7 (65.19 [25.55]). Similar outcomes in mean IUS scores were observed in an LOCF analysis $\left(t_{25}=4.46, P<.0001\right.$, Cohen $d=0.66$ ), where mean (SD) IUS scores decreased significantly from visit $1(81.32$ [18.22] $)$ to visit 7 (66.71 [25.21]). Significance was not observed in an OC complete analysis of BAI scores from visit 1 to visit $7\left(t_{25}=0.022, P=.983\right)$. There was a small difference in mean (SD) BAI scores from visit 1 (12.88 [8.89]) to visit 7 (12.84 [11.36]). Similar outcomes were observed in the LOCF analysis $\left(t_{25}=-0.19\right.$, $P=.849$ ), where mean (SD) BAI scores did not differ significantly from visit $1(12.93$ [8.56]) to visit 7 (13.25 [11.03]). Table 1 and Table 2 summarize the results.

\section{OMTh Findings}

The black-box study design requires an individualized approach to OMTh for each participant; thus no 2 sessions were identical. However, some common structures were treated among the study sample, which became apparent after completion of the study (Table 3).

\section{Discussion}

This study investigated the efficacy of using OMTh to treat participants with moderate to severe GAD. Overall, the results were promising because patients who received 5 sessions of OMTh over a period of 8 to 9 weeks demonstrated a significant reduction, and in

\section{Table 1. \\ Observed Case Analysis Scores of Participants With Moderate to Severe General Anxiety Disorder Before and After Osteopathic Manipulative Therapy ${ }^{a}(\mathrm{~N}=26)$}

\begin{tabular}{lcc}
\multirow{2}{*}{ Outcome Measures } & \multicolumn{2}{c}{ Score, Mean (SD) } \\
\cline { 2 - 3 } & Visit 1 & Visit 7 \\
\hline HAM-A & $26.19(3.88)$ & $11.92(6.11)$ \\
\hline BAI & $12.88(8.89)$ & $12.84(11.36)$ \\
\hline IUS & $80.08(18.3)$ & $65.19(25.6)$ \\
\hline
\end{tabular}

a Manipulative care provided by foreign-trained osteopaths. Five 60-minute sessions were provided over 8 weeks.

Abbreviations: BAI, Beck Anxiety Inventory; HAM-A, Hamilton Anxiety Rating Scale; IUS, Intolerance for Uncertainty Scale.

some cases, complete remittance, in explicit and implicit symptoms associated with GAD. These findings suggest that OMTh may be a useful adjunctive treatment to standard care in alleviating distress associated with GAD and may contribute to better outcomes from psychotherapeutic and pharmacologic interventions.

The results from this investigation indirectly highlight the distinction between the cognitive and physical symptoms of GAD, which can be inferred from the differences in results. The significant score reductions on both the HAM-A and the IUS were observed, but

Table 2.

LOCF Analysis Scores of Participants With Moderate to Severe GAD Before and After Osteopathic Manipulative Therapy ${ }^{a}(\mathbf{N}=26)$

\begin{tabular}{ccc}
\multirow{2}{*}{\begin{tabular}{c} 
Lutcome Measures \\
\cline { 2 - 3 }
\end{tabular}} & Visit 1 & Visit 7 \\
\hline HAM-A & $26.25(3.74)$ & $12.39(6.14)$ \\
\hline BAI & $12.93(8.56)$ & $13.25(11.03)$ \\
\hline IUS & $81.32(18.22)$ & $66.71(25.21)$ \\
\hline
\end{tabular}

a Manipulative care provided by foreign-trained osteopaths. Five 60 -minute sessions were provided over 8 weeks.

Abbreviations: BAI, Beck Anxiety Inventory; HAM-A, Hamilton Anxiety Rating Scale; IUS, Intolerance for Uncertainty; LOCF, last observed visit carried forward. 


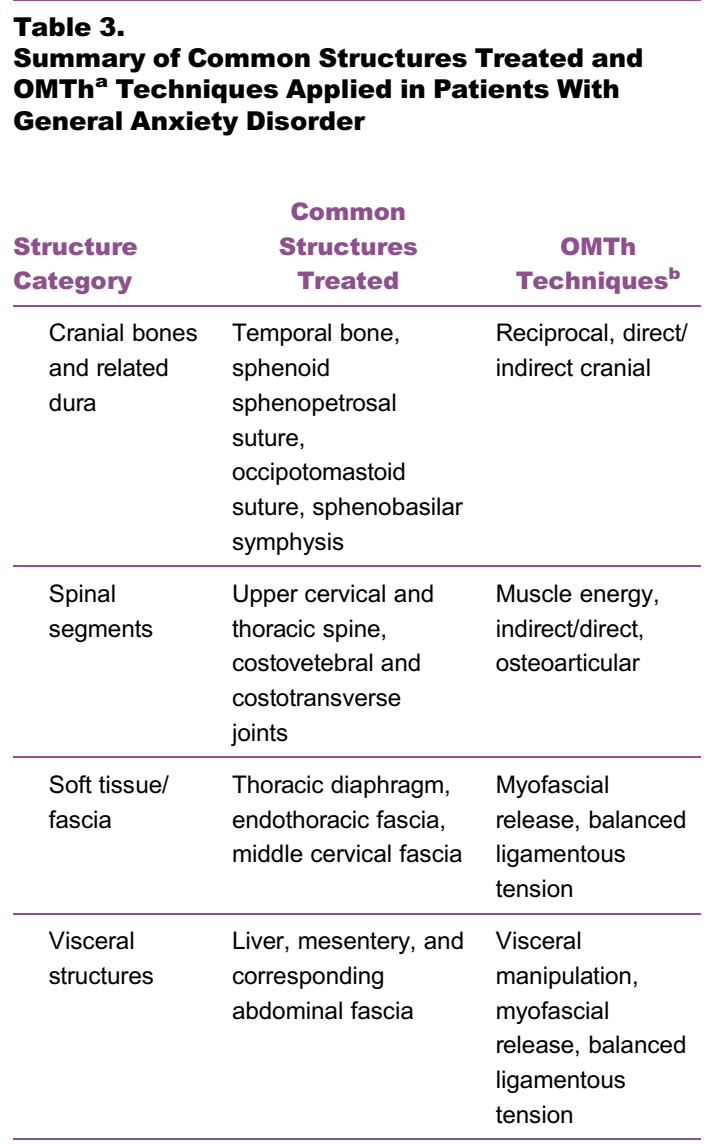

a Manipulative care provided by foreign-trained osteopaths. Five 60-minute sessions were provided over 8 weeks.

b The type of osteopathic manipulative therapy (OMTh) technique used was dependent on the individual patient.

scores on the BAI did not change significantly. We propose that the difference in scores after OMTh may reflect the differences in measured features of anxiety between the HAM-A, IUS, and BAI. For example, the HAM-A and IUS constructs may be more concerned with cognitive symptoms of anxiety, as opposed to the BAI, which is concerned with the physiologically experienced symptoms during an anxiety attack. ${ }^{35,37}$ Furthermore, the degree and type of comorbidities observed in this population may also bear implications about the differences observed.

Both the HAM-A and the IUS assessed severity of cognitive symptoms characteristic of GAD, whereas the BAI more closely assessed discomfort or fear associated with the experience of physical symptoms of anxiety. ${ }^{35,37}$ The HAM-A contained items that required elaboration and warranted further discussion between the participant and physician, which could potentially yield more accurate ratings. Despite being self-reported, the IUS also contained longer and more specific statements that participants were required to rate, whereas the BAI contained only a few adjectives that described distress from physical sensations. In part, the physical anxiety symptoms were likely more characteristic of a panic disorder presentation, hence why they may not have been as salient in this GAD population. ${ }^{39}$ In this way, perhaps OMTh helped improve cognitive symptoms of GAD, including the inability to tolerate uncertainty, rather than the concerns about the physical symptoms of anxiety. ${ }^{2}$

An alternative explanation may be that BAI scores in this particular cohort were relatively low at visit 1 , which suggests a possible floor effect with scores nearing the lower limit on the BAI, thus preventing observable variability. ${ }^{50}$ Moreover, participants may have scored lower on the baseline BAI in anticipation of the OMTh that they would receive and hoped that it would help them feel better, ${ }^{39}$ thereby biasing their reports.

Another compelling explanation for differences observed in the results may be that both the IUS and HAM-A correspond to and predict the comorbid conditions represented in the study sample. ${ }^{42,45}$ A high percentage $(92.3 \%)$ of this sample had concurrent major depressive disorder, which is the most common comorbid disorder associated with GAD according to the literature. ${ }^{2}$ The shared improvement in symptoms observed in the scores on both the HAM-A and the IUS support the literature that demonstrates a strong correlation between baseline scores on both of these constructs. $^{42,45}$ However, it should be noted that the HAM-A has been criticized for its lack of specificity, or discriminant validity, because of the overlap with other mood disorders, including depression. ${ }^{39}$ Conversely, the BAI has been criticized for content 
validity related to the exclusion of depression symptoms. ${ }^{39}$ This controversy may therefore support the need for a transdiagnostic approach to understanding and treating mental disorders in order to yield better outcomes.

From an osteopathic perspective, the body-mind interrelationships and the effect on the autonomic nervous system may also serve as a partial explanation for these results. ${ }^{7,51,51}$ Specifically, OMTh appears to decrease sympathetic tone $\mathrm{e}^{52}$ by improving mobility of the ribs and spine in the region of the sympathetic ganglions. It may also help facilitate parasympathetic tone ${ }^{52}$ by decreasing tension at the cranial base, the region where the cranial nerves are located, ${ }^{14}$ and the fascial paths of vagus nerve branches, as well as the sacrum. Furthermore, decreasing muscle and fascial tension may affect afferent feedback to the brain and HPA axis output. ${ }^{7,13,52}$ It has also been established that therapeutic touch contributes to the release of oxytocin, and because touch is an integral part of osteopathic care through gentle manual manipulations, it can be inferred that OMTh may boast similar effects. ${ }^{12}$ Ensuring that segments and fascia related to the gut are free of tension may also indirectly influence the brain via the gut-brain theory. ${ }^{20}$ In its approach of treating the whole person, osteopathic philosophy recognizes that treating one area of the body or system can ultimately affect another. ${ }^{8}$

General anxiety disorder is a highly comorbid, complex condition and is systemic in its nature, affecting multiple systems of the body. ${ }^{2}$ Osteopathic manipulative therapy addresses this complexity with holistic and multifaceted approach, respecting the functional unity of the body. ${ }^{8,9}$ It considers compromised body functions as important to understanding the overall picture of the patient's presenting condition. Each compromised state, for example, impaired digestion, increased pain, disturbed sleep-wake cycle, inhibited immune function, and increased muscle tension can affect or even induce another compromised state. Each dysfunctional state can also activate the HPA axis and ultimately influence the brain and a person's emotional response. $^{7}$
Limitations must be considered in the interpretation of the data. Our study lacked a comparison group that did not receive OMTh; therefore, it is difficult to determine whether the positive results were due to the nonspecific physiological effects of touch. ${ }^{11,53}$ Without an active control group, it can be argued that OMTh expectation effects may have confounded the results. ${ }^{53}$

Additionally, our study neither controlled for factors including diet and physical activity level, nor controlled for the various patient-clinician effects. ${ }^{11,53}$ Lack of blinding of the clinicians and the open-label design may have resulted in bias, including demand characteristics. $^{54}$ Specifically, knowing that the clinicians believed that the OMTh might help may have influenced outcome measure results, with participants subconsciously feeling pressured to indicate improvements on outcome measures. ${ }^{37,53}$ However, this factor may not have had a significant effect on the results given that some outcome measurements (specifically the BAI) did not improve.

Trust or hope that may have been established through the therapeutic relationship itself may have contributed to positive effects observed during OMTh. ${ }^{12,53}$ However, because physical contact and the therapeutic relationship are integral to OMTh and its efficacy, separating these aspects of OMTh may be unnecessary and not conducive in determining its efficacy. Future studies should consider using a double-blind crossover design and a comparison control group as well as longer-term follow-up to chart the course of this adjunctive treatment approach for patients with GAD.

\section{Conclusion}

The purpose of this study was to determine whether OMTh had a positive effect on symptoms related to GAD. By demonstrating significant reductions in symptoms of anxiety (as measured by the HAM-A and IUS), this study provides preliminary evidence for the use of OMTh as an adjunctive treatment for GAD. The openlabel, black-box, before-after study design limits any definitive clinical recommendations and replication. 
However, it has the advantage of reflecting the actual holistic practice of OMTh in its consideration for the individual. As such, this study has provided a platform and rationale for further research concerning body-mind interaction. The present study highlights an approach that may help increase awareness and discussion around an integrative model in the adjunctive use of OMTh in the management of GAD.

\section{Author Contributions}

All authors provided substantial contributions to conception and design, acquisition of data, or analysis and interpretation of data; all authors drafted the article or revised it critically for important intellectual content; all authors gave final approval of the version of the article to be published; and all authors agree to be accountable for all aspects of the work in ensuring that questions related to the accuracy or integrity of any part of the work are appropriately investigated and resolved.

\section{References}

1. Walker ER, McGee RE, Druss B. Mortality in mental disorders and global disease burden implications: a systematic review and meta-analysis. JAMA Psychiatry. 2015;72(4):334-341. doi:10.1001/ jamapsychiatry.2014.2502

2. Katzman MA, Bleau P, Blier $P$, et al. Canadian clinical practice guidelines for the management of anxiety, posttraumatic stress and obsessive-compulsive disorders. BMC Psychiatry. 2014;14(suppl 1): S1. doi:10.1186/1471-244X-14-S1-S1

3. Newman MG, Llera SJ, Erickson TM, Przeworski A, Castonguay LG Worry and generalized anxiety disorder: a review and theoretical synthesis of evidence on nature, etiology, mechanisms, and treatment. Annu Rev Clin Psychol. 2013;9:275-297. doi:10.1146/ annurev-clinpsy-050212-185544

4. Nepon J, Belik SL, Bolton J, Sareen J. The relationship between anxiety disorders and suicide attempts: findings from the National Epidemiologic Survey on Alcohol and Related Conditions. Depress Anxiety. 2010;27(9):791-798.

5. Cuijpers P, Sijbrandij M, Koole S, Huibers M, Berking M, Andersson G. Psychological treatment of generalized anxiety disorder: a meta-analysis. Clin Psychol Rev. 2014;34(2):130-140. doi:10.1016/j.cpr.2014.01.002

6. Ravindran AV, da Silva TL. Complementary and alternative therapies as add-on to pharmacotherapy for mood and anxiety disorders: a systematic review. J Affect Disord. 2013;150(3):707-719. doi:10.1016/j. jad.2013.05.042

7. Taylor AG, Goehler LE, Galper DI, Innes KE, Bourguignon C. Top-down and bottom up mechanisms in mind-body medicine: development of an integrative framework for psychophysiological research. Explore. 2010;6(1):29-41. doi:10.1016/j.explore.2009.10.004

8. Seffinger MA, King HH, Ward RC, Jones JJ, Rogers FJ, Patterson MM. Osteopathic philosophy. In: Chila AG, ed. Foundations of Osteopathic Medicine. 3rd ed. Baltimore, MD: Lippincott Williams \& Wilkins; 2011.
9. Still AT. Philosophy and Mechanical Principles of Osteopathy. Kansas City, MO: Hudson-Kimberly Pub Co; 1902.

10. Elkiss ML, Jerome JA. Touch-more than a basic science. J Am Osteopath Assoc. 2012;112(8):514-517.

11. Ellingsen DM, Siri Leknes S, Løseth G, Wessberg J, Olausson H. The neurobiology shaping affective touch: expectation, motivation, and meaning in the multisensory context. Front Psychol. 2015;6:1986 doi:10.3389/fpsyg.2015.01986

12. Uvnas-Moberg K, Petersson M. Role of oxytocin and oxytocin related effects in manual therapy. In: King N, Janig W, Patterson MM. The Science and Clinical Application of Manual Therapy. London, England: Churchill Livingstone; 2011:147-162.

13. Tozzi, P. Selected fascial aspects of osteopathic practice. J Bodyw Mov Ther. 2012;16(4):503-519. doi:10.1016/j.jbmt.2012.02.00

14. Wilson-Pauwels L, Stewart PA, Akesson EJ, Spacey SD. Vagus nerve In: Cranial Nerves Function \& Dysfunction. 3rd ed. Shelton, CT: People's Medical Publishing House; 2010:188-201.

15. Patterson MM, Wurster RD. Somatic dysfunction, spinal facilitation, and viscerosomatic integration. In: Chila AG, ed. Foundations of Osteopathic Medicine. Baltimore, MD: Lippincott Williams \& Wilkins; 2011:118-133.

16. Adstrum S, Hedley G, Schleip R, Stecco C, Yucesoy CA. Defining the fascial system. J Bodyw Mov Ther. 2017;21(1):173-177. doi:10.1016/j. jbmt.2016.11.003

17. Willard FH, Fossum C, Standley PR. The fascial system of the body. In: Chila AG,ed. Foundations of Osteopathic Medicine. Baltimore, MD: Lippincott Williams \& Wilkins; 2011:74-92.

18. O'Donovan A, Hughes BM, Slavich GM, et al. Clinical anxiety, cortisol and interleukin-6: evidence for specificity in emotion-biology relationships. Brain, Behav Immun. 2010;24(7):1074-1077. doi:10.1016/j.bbi.2010.03.003

19. Fond G, Loundou A, Hamdan N, et al. Anxiety and depression comorbidities in irritable bowel syndrome (IBS): a systematic review and meta-analysis. Eur Arch Psychiatry Clin Neurosci. 2014;264 (8):651-660. doi:10.1007/s00406-014-0502-z

20. Dinan TG, Cryan JF. Regulation of the stress response by the gut microbiota: implications for psychoneuroendocrinology. Psychoneuroendocrinology. 2012;37(9):1369-1378. doi:10.1016/j. psyneuen.2012.03.007

21. Barral JP, Mercier P. Visceral manipulation (Revised edition). Seattle, WA: Eastland Press; 2007.

22. Shi X, Rehrer S, Prajapati P, Stoll ST, Gamber RG, Downey HF. Effect of cranial osteopathic manipulative medicine on cerebral tissue oxygenation. J Am Osteopath Assoc. 2011;111(12):660-666.

23. Ponzo V, Cinnera AM, Mommo F, Catagirone C, Koch G, Tramontano M. Osteopathic manipulative therapy potentiates motor cortical plasticity. J Am Osteopath Assoc. 2018;118(6):396-402: doi:10.7556/ jaoa.2018.084

24. Walkowski S, Singh M, Puertas J, Pate M, Goodrum K, Benencia F. Osteopathic manipulative therapy induces early plasma cytokine release and mobilization of a population of dendritic cells. PLoS One. 2014;9(3):e90132. doi:10.1371/journal.pone.0090132

25. Henley CE, Ivins D, Mills M, Wen FK, Benjamin BA. Osteopathic manipulative treatment and its relationship to autonomic nervous system activity as demonstrated by heart rate variability: a repeated 
measures study. Osteopath Med Prim Care. 2008;2(7). doi:10.1186/ $1750-4732-2-7$

26. Ajimsha MS, Al-Mudahka NR, Al-Madzhar JA. Effectiveness of myofascial release: systematic review of randomized controlled trials. J Bodyw Mov Ther. 2015;19(1):102-112. doi:10.1016/j.jbmt.2014.06.001

27. Voigt K, Liebnitzky J, Burmeister U, et al. The efficacy of osteopathic manipulative treatment of female patients with migraine: results of a randomized controlled trial. J Altern Complement Med. 2011;17 (3):225-230. doi:10.1089/acm.2009.0673

28. D'Ippolito M, Tramontano M, Buzzi MG. Effects of osteopathic manipulative therapy on pain and mood disorders in patients with high-frequency migraine. J Am Osteopath Assoc. 2017;117 (6):365-369: doi:10.7556/jaoa.2017.074

29. Cicchitti L, Martelli M, Cerritelli F. Chronic inflammatory disease and osteopathy: a systematic review. PLoS ONE. 2015;10(3):e0121327. doi:10.1371/journal.pone.0121327

30. Verhaeghe N, Schepers J, van Dun P, Annemans L. Osteopathic care for spinal complaints: a systematic literature review. PLOS ONE. 2018;13(11):e0206 doi:10.1371/journal.pone.0206284284

31. Plotkin BJ, Rodos JJ, Kappler R, et al. Adjunctive osteopathic manipulative treatment in women with depression: a pilot study. J Am Osteopath Assoc. 2001;101(9):517-522.

32. Cutler MJ, Holland BS, Stupski BA, Gamber RL, Smith ML. Cranial manipulation can alter sleep latency and sympathetic nerve activity in humans: a pilot study. J Altern Complement Med. 2005;11(1):103-108. doi:10.1089/acm.2005.11.103

33. Espí-López GV, López-Bueno L, Vicente-Herrero MT, Martinez-Arnau FM, Monzani L. Efficacy of manual therapy on anxiety and depression in patients with tension-type headache: a randomized controlled trial. Int J Osteopath Med. 2016;22:11-20. doi:10.1016/j.ijosm.2016.05.003

34. Sheehan DV, Lecrubier $\mathrm{Y}$, Sheehan $\mathrm{KH}$, et al. The Mini-International Neuropsychiatric Interview (M.I.N.I.): the development and validation of a structured diagnostic psychiatric interview for DSM-IV and ICD-10. J Clin Psychiatry.1998;59(suppl 20):22-33.

35. Hamilton M. The assessment of anxiety states by rating. $\mathrm{Br} \mathrm{J}$ Med Psychol. 1959;32(1):50-55. doi:10.1111/j.2044-8341.1959.tb00467.x

36. Hamilton M. A rating scale for depression. J Neurol Neurosurg Psychiatry. 1960;23:56-62.

37. Beck AT, Steer RA. Manual for the Beck Anxiety Inventory. San Antonio, TX: Psychological Corporation; 1990

38. Buhr K, Dugas MJ. The intolerance of uncertainty scale: psychometric properties of the English version. Behav Res Ther. 2002;40 (8):931-945. doi:10.1016/s0005-7967(01)00092-4

39. McDowell I. Measuring Health. 3rd ed. New York, NY: Oxford University Press; 2006

40. Doyle AC, Pollack MH. Establishment of remission criteria for anxiety disorders. J Clin Psychiatry. 2003;64(suppl):40-45.

41. Beck AT, Epstein N, Brown G, Steer RA. An inventory for measuring clinical anxiety: psychometric properties. J Consult Clin Psychol. 1988;56(6):893-897. doi:10.1037//0022-006x.56.6.893
42. Gentes EL, Ruscio AM. A meta-analysis of the relation of intolerance of uncertainty to symptoms of generalized anxiety, major depressive disorder, and obsessive-compulsive disorder. Clin Psychol Rev. 2011;31(6):923-933. doi:10.1016/j.cpr.2011.05.001

43. Burh K, Dugas M. Investigating the construct validity of intolerance of uncertainty and its unique relationship with worry. J Anxiety Disord. 2006;20(2):222-236. doi:10.1016/j.janxdis.2004.12.004

44. McEvoy PM, Mahoney AE. Achieving certainty about the structure of intolerance of uncertainty in a treatment-seeking sample with anxiety and depression. J Anxiety Disord. 2011;25(1):112-122. doi:10.1016/J. janxdis.2010.08.010

45. Boswell JF, Thompson-Hollands J, Farchione TJ, Barlow DH. Intolerance of uncertainty: a common factor in the treatment of emotional disorders. J Clin Psychol. 2013;69(6):630-645. doi:10.1002 jclp.21965

46. Osteopathic assessment and treatment. Canadian College of Osteopathy website. http://www.osteopathy-canada.com/w osteopathic-manual-practitioner-canada.aspx? Accessed January 7 , 2020

47. Magoun H. Osteopathy in the Cranial Field. 3rd ed. Kirksville, MO: The Journal Printing Company; 1976.

48. Franke H, Franke JD, Fryer G. Osteopathic manipulative treatment for chronic nonspecific neck pain: a systematic review and meta-analysis. Int J Osteopath Med. 2015;18(4):255-267. doi:10.1016/j. ijosm.2015.05.003

49. Godwin M, Ruhland L, Casson I, et al. Pragmatic controlled clinical trials in primary care: the struggle between external and internal validity. BMC Med Res Methodol. 2003;3:28. doi:10.1186/ 1471-2288-3-28

50. Vogt WP, Johnson RB, eds. Dictionary of Statistics \& Methodology: A Nontechnical Guide for the Social Sciences. 4th ed. Newbury Park, CA: Sage; 2011

51. Baron DA, Julius, RJ, Willard, FH. Psychoneuroimmunology-basic mechanisms. In: Chila AG, ed. Foundations of Osteopathic Medicine. 3rd ed. Baltimore, MD: Lippincott Williams \& Wilkins 2011:276-283.

52. Fornari M, Carnevali L, Sgoifo A. Single osteopathic manipulative therapy session dampens acute autonomic and neuroendocrine responses to mental stress in healthy male participants. $J \mathrm{Am}$ Osteopath Assoc. 2017;117(9):559-567. doi:10.7556/jaoa.2017.110

53. Patterson MM. The use of sham or placebo controls in manual medicine research. In: King H, Janig W, Patterson MM, eds. The Science and Clinical Application of Manual Therapy. London, England: Churchill Livingstone; 2011:181-191.

54. Nichols AL, Maner JK. The good subject effect: investigating participant demand characteristics. J Gen Psychol. 2008;135 (2):151-165. doi:10.3200/GENP.135.2.151-166

55. White A, Ernst E. The case for uncontrolled clinical trials: a starting point for the evidence based for CAM. Complement Ther Med. 2001;9 (2):111-116. doi:10.1054/ctim.2001.0441

(2) 2020 American Osteopathic Association 